\title{
Signal Cascades Analysis in Nanoprocesses with Distributed Database System
}

\author{
Dariusz Mrozek, Bożena Małysiak, Jacek Frączek, and Paweł Kasprowski \\ Silesian University of Technology, Department of Computer Science, \\ ul. Akademicka 16, 44-100 Gliwice, Poland \\ \{Mrozek, malysiak, kasprowski\}@polsl.pl, \\ jacekf@polsl.gliwice.pl
}

\begin{abstract}
The signal cascades are a number of successive biochemical reactions, occurring in the cells. In these reactions take part many proteins (often enzymes) and the entire process may be compared to the dominoes effect. The common term used for define a varied biochemical mechanisms regulating processes in the nanonetworks is signal transduction executed in the signal cascades. These processes can be realized in a closed area of space which contains proper quantities of substrates and a set of control molecules working in a predefined manner which was determined by their chemical construction, including changes of chemical activity reached usually by the conformational changes. Information about the signal cascades that happen in the various type of cells for given processes can be retrieved from the biochemical research accessible in the biochemical databases. In this work, the simulation process of the signal transduction in bio-nanoprocesses using the distributed database environment is presented and illustrated by the biological example.
\end{abstract}

\section{Introduction}

In outward things development of nanotechnology is in initial stage. Nowadays, manufacturing methods are very simple and basic at the molecular level. Manufactured products are made from cells. The properties of those products depend on how those cells are arranged. The first approach to implement the nanoprocesses would be coping biocompatible standards in design and implementation phase of such processes. The signal cascades are a number of successive biochemical reactions, occurring in the cells. In these reactions take part many proteins (often enzymes) and the entire process may be compared to the dominoes effect - when the first protein of the cascade is modified, such modified protein has an effect on the next protein modifying it in some direction. Thanks to the signal cascades diverse organisms can live and develop. Furthermore, if some protein take part in signal cascade incorrectly (as a result of mutation in gene coding this protein), effect of this single change can result in the tumor development.

The common term used for define a varied biochemical mechanisms regulating processes in the nanonetworks is signal transduction which is executed in the signal cascades. These processes (coping biocompatible solutions) can be realized in a closed area of space which contains appropriate quantities of substrates and a set of con- 
trol molecules working in a predefined manner which was determined by their chemical construction, including changes of chemical activity reached usually by the conformational changes. These molecules have a decisive role of a control system. The system is stimulated by external signal molecules coming from outside of the closed area of space to its surface, meeting the function of nanoprocess border. The signal cascades are the fixed cycles of transformations in which the molecular control system performs its control tasks. Actually, this kind of system performing nanoprocess ought to be called as a nanonetworks. The selected substrates (inorganic ions and most of metabolites e.g. sugars, amino acids, and nucleotides) and final products can penetrate through the border of the process in determined conditions. The external stimulating signal molecules only activate the receptors placed in the border of a nanonetwork, excluding selected ions fulfilling the control functions in the signal cascades. The signal transduction $[4,21]$ is the process of internal transformations and conversions of control molecules in signal cascades expressed as a control of nanoprocesses in a nanonetwork. Generally, one can talk about wide area and local nanonetworks. In living organisms wide area nanonetworks $[13,19]$ is represented by an electro-chemical net (e.g. nervous system) and extracellular communication net (e.g. immune and hormone systems). The local area nano-network is represented by a cell. Exploitation of a living cell is one of the most natural (but not basic) approach to build the local nanonetwork performing a given production process, which can be modified through the external control. An assurance of survival requirements is the basic condition, which have to be satisfied for the cell. Stimulation of inputs of the signal cascades in a nanoprocess cell from its environment is necessary for the control. Information about the signal cascades that happen in the various type of cells for given processes can be retrieved from the biochemical research available in the biochemical databases. Finally, a sequence of stimuli signals for given process can be determined.

\section{Architecture of the Simulation System Implementation}

The mirror-based architecture presented in Fig. 1 defines the foundation of the simulation system used in the signal cascade analysis. The approach arose as the consequence of the distribution of domain-specific information. Nowadays, the growing number of central repositories for collecting biochemical and biomedical data is observed and many organizations, research institutes and university laboratories around the world lead their projects in order to understand the mysterious nature of living cells. This cause the situation the particular information is distributed in many databases managed by different institutions connected to specific domain, e.g. the huge amount of biomolecular structural data is stored in public databases of the PDB (Protein Data Bank [1]) managed by the RCSB ${ }^{1}$ or NCBI's ${ }^{2}$ MMDB [20], amino acid sequence information stored in the UniProt ${ }^{3}[3,11]$ and NCBI's databases, human gene data stored in the H-Invitational DB provided by JBIRC ${ }^{4}$, protein interaction data

\footnotetext{
${ }^{1}$ Research Collaboratory for Structural Bioinformatics (http://www.rcsb.org).

${ }^{2}$ National Center for Biotechnology Information (http://www.ncbi.nlm.nih.gov).

${ }^{3}$ UniProt Consortium (http:// www.uniprot.org).

${ }^{4}$ Japan Biological Information Research Center (http://www.jbirc.aist.go.jp).
} 
managed by BIND $^{5}$ [12], etc. Fortunately for the community, all the data is available from these institution's FTP sites in the form of text files (e.g. PDB [17], mmCIF [5] formats), XML-structured files or using dedicated application programming interfaces (OMG's Macromolecular Structure Specification and Biomolecular Sequence Analysis Specification [15]) and loaders (OpenMMS [2, 18] toolkit). Searching information across distributed heterogeneous database systems that store all the enormous volumes of data has become a very important aspect of the scientific research and may be a great challenge.

In the mirror-based approach in Fig. 1, during the query process, data does not come directly from the distributed data sets. The complete sets or subsets of databases are first mirrored to the local server(s) and then queries are submitted to local replicas. In this architecture the source databases are called the primary databases and the local servers are called the secondary databases.

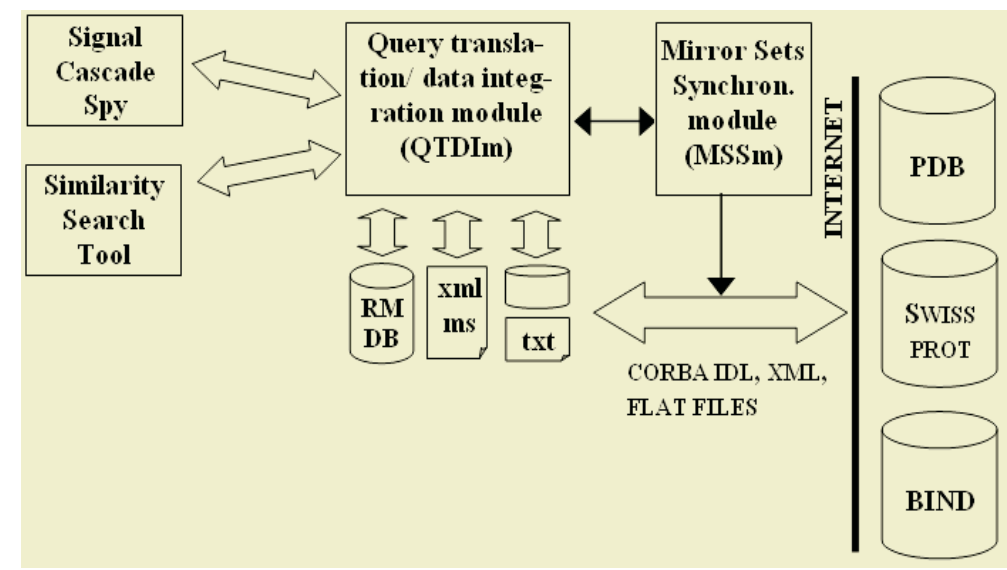

Fig. 1. The architecture with mirrored data sets synchronized with the primary databases

The main components of the architecture are: mirrored datasets in the form of relational databases (RMDB icon - Relational Mirrored Database), XML- and TXT- format mirror sets (XML-MS, TXT), the Query Translation/Data Integration module (QTDIm), the Mirror Sets Synchronization module (MSSm) and End-User tools. User queries, similarity searching and advanced computing are triggered from End-User Tools through the QTDIm which is a kind of controller in the process of information extraction. The synchronization process is coordinated by the MSSm, which can be forced to make replicas by the QTDIm, if needed.

\section{Approximate Methods of Similarity Searching}

At present, the afford of implementing the structure similarity search has been made with approximate methods. The main assumption of approximate retrieval methods is

\footnotetext{
${ }^{5}$ Biomolecular Interaction Network Database of Blueprint Initiative (http://www.bind.ca).
} 
that for the answer on the given query we can obtain a set of the objects from database which are consistent with criteria defined in the query with given degree. Queries of this type require defining characteristic function, which determines in what degree the searched object is consistent with criteria defined in the query and threshold value, which allows to qualify the objects that should occur in the answer. Existing retrieval algorithms for the biological databases are based on principles valid in the objects approximate retrieval methods. In the biological databases two trends are separated:

- similarity searching by a protein sequences alignment,

- similarity searching by alignment of a three-dimensional protein structures.

During the research (example introduced in section 5) the PDB [1], BIND [12] and BioCarta [9] datasets were exploited but there is a possibility to access other mirrored databases like UniProt/Swissprot [11] and GenBank [8]. Some features of developed simulation software are presented in Fig. 2.

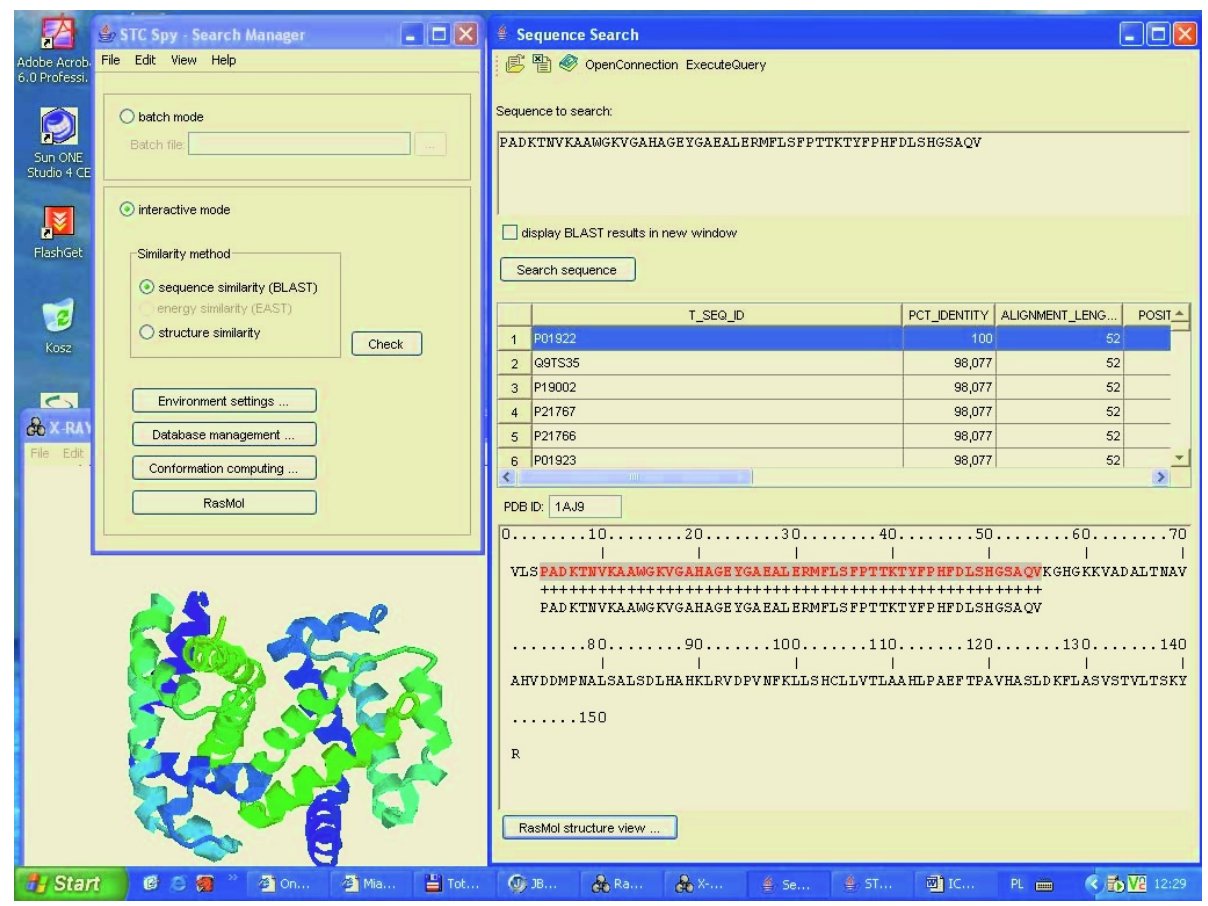

Fig. 2. Signal Transduction Spy - the Search Manager

Two modes of preparing experiment remain feasible: batch mode and interactive step-by-step mode (left window). Processing data in the batch mode seems to be better because it is going without any control and doesn't affect the user attention - the experiment may take a long time especially during all the processes that use protein conformation computation module to investigate changes in protein 3D structure as a result of environmental parameters changes (e.g. temperature, $\mathrm{pH}$ ). However, the batch process is supposed to be described in the special purpose process-description files containing all steps of the computation. The mode is in the development phase. 
The interactive mode provides users to see what changed during each step of the experiment and to make corrections or to change the way the process goes (e.g. see each step of the signal transduction and the substrates of the cascade, see the network of interactions, explore of protein conformation, change the similarity search method, etc.). In the mode users make use of RasMol [10] to display semi-result structures of their activities in graphical form.

\section{Processing of a Single Step of the Signal Cascade}

Processing of the signal cascade may be divided on processing respective steps of the cascade, represented by reactions on each level. Fig. 3a presents a sample network of the reactions between nanostructures (e.g. proteins, polypeptides). Each reaction results with a creation of a final product. The product may be a protein in the appropriate state and conformation, which can be the activator or inhibitor of the other reactions which take place in the living cell or nanoprocess. Fig. $3 \mathrm{~b}$ shows the algorithm of tracking the signal cascade step - e.g. 1.7.2.24 reaction [24] marked in the Fig. 3a.
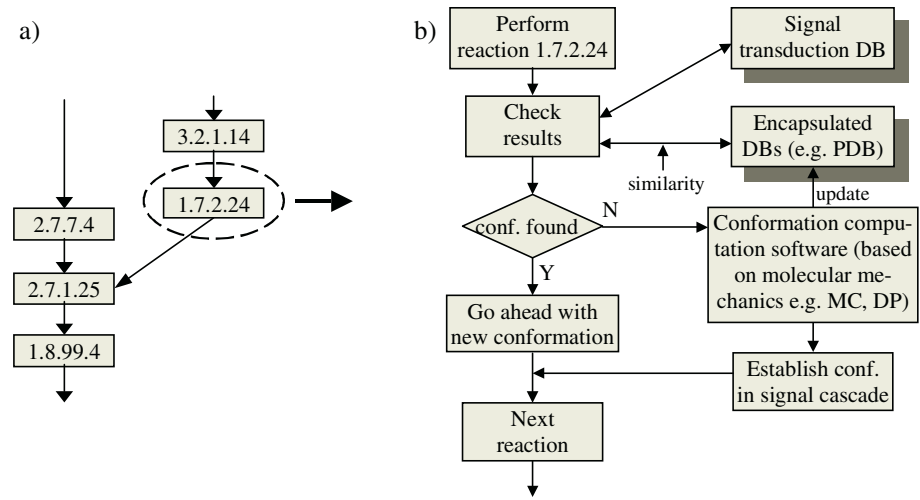

Fig. 3. a) Sample network of reactions, b) Processing of a single step of the cascade

During the process of tracking cascade's steps it is possible to check the conformational results (of nanostructure like polypeptide) with the databases consisting of the structural information (e.g PDB [1]). These databases work as an integral parts of the entire system. The retrieval of similar polypeptides is based on the approximate methods (section 3). If the structure or similar structure (with similarity determined) is found in the database, the system retrieves appropriate information and tracks to the next step of the cascade with the retrieved polypeptide. The information about cascade steps comes from other databases (e.g. BioCarta [9], BIND [12], aMAZE [24]). If the resultant structure is not found in the database, it must be computed with the use of molecular mechanics methods and software, with the assumption of getting minimal potential energy. We are strongly convinced that minimizing of the energy expression describing a conformational energy of structure [7, 22, 23] leads to the problem related with multiple local minima or saddle points and so simple algorithms of optimization e.g. gradient-based, Newton's or Fletcher-Powell have to be modified to find 
the global minimum. The implementation of algorithms of dynamic programming (DP) [25] or Monte Carlo (MC) methods [16, 26] is better solution. Moreover, these methods are time- and computer's memory consuming. Once the new conformation is established, it is possible to look for the interactions with other proteins and reactions the new protein can participate and meanwhile the database is being updated.

\section{Example of Selected Process in the Signal Cascade Analysis}

The dataflow in the signal transduction simulation system using the distributed databases environment [14] is presented in Fig. 4. In the system the three databases work as encapsulated parts of the computing process visible through the interfaces $\mathrm{A}$ and $\mathrm{B}$.

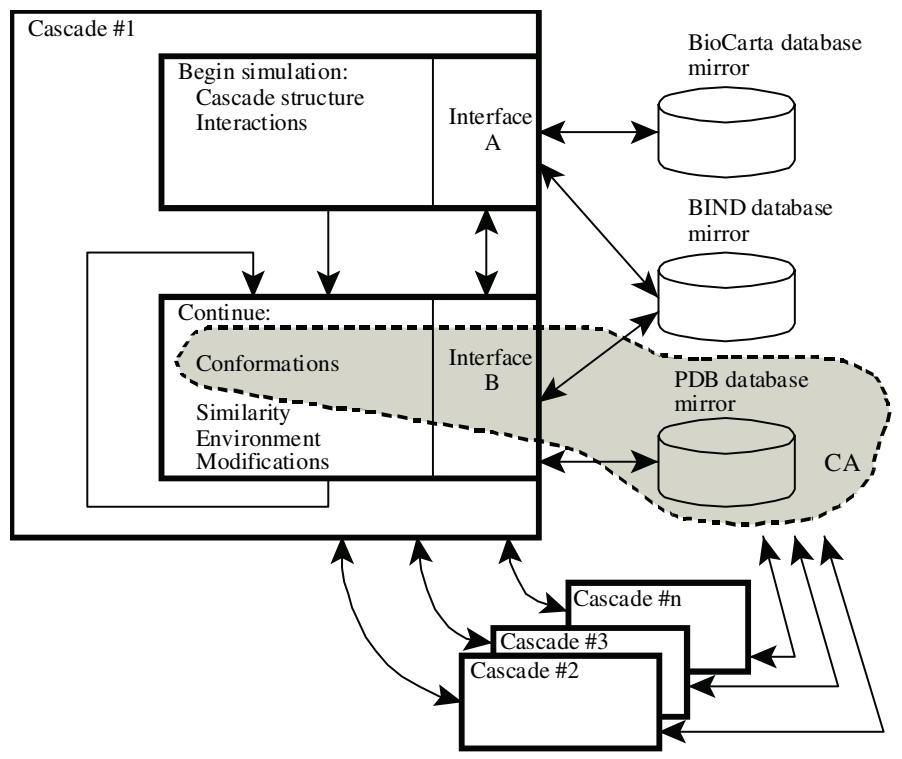

Fig. 4. Dataflow in simulation process

The BioCarta database mirror allows the cascade structures data [9], the BIND database mirror allows nanostructures interactions data [12], and the PDB database mirror allows the molecule structures data [1].

The example presents the approach of conformation determination in signal cascade including the human CDK2 kinase during the phosphorylation process [6] using proper database. In inactive form the CDK2 kinase can be retrieved from the PDB database as molecule signed by 1B38 [1]. During the phosphorylation process the threonine 160 in main chain of CDK2 is modified. To find this conformation change is very difficult by computation. The better solution is to search the proper, existing information from database. In Fig. 4 the shadowed area CA presents this simulation/database operation. To make more visible the critical place responsible for the conformation of kinase CDK2 (in the ATP complex) described by the 1B38 file was processed to extract the threonine 160 neighborhood [6]. Retrieved from 1B38 file the middle 
part of the main chain of amino acids of the CDK2 is presented in Fig. 5a. The same part of the main chain of CDK2 after the phosphorylation (extracted from 1B39 file from PDB database) is presented in Fig. 5b. The changes in the conformation can be found basing on molecular mechanics approach, however, the results obtained from operation CA using selected database seem to be more practical.

a)

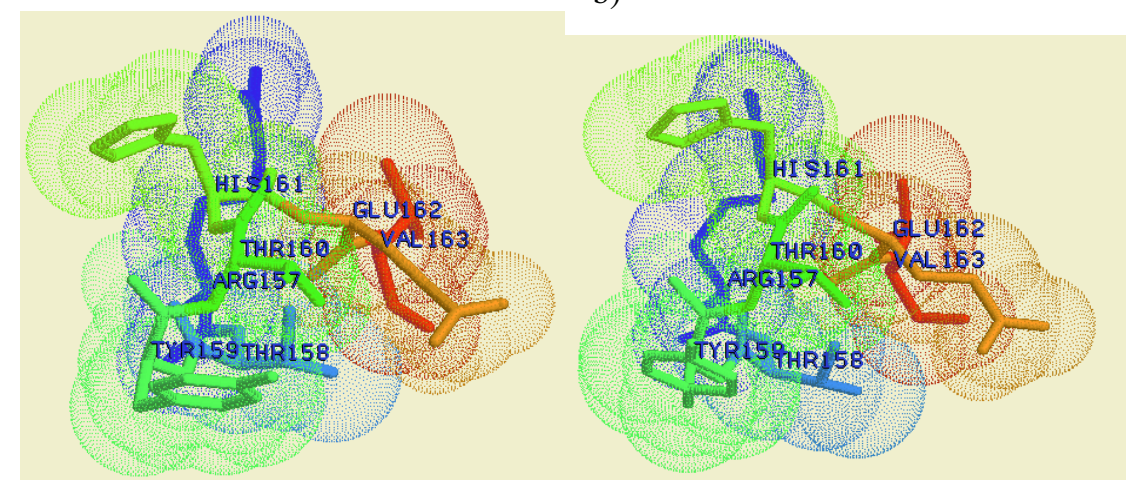

Fig. 5. a) Sticks representation with the van der Waal's dot surfaces of the conformation in a middle part (residues 157-163) of the non-phosphorylated CDK2 (the threonine 160 in the center of view), b) the same view of CDK2 in a middle part of the amino acids chain (residues 157-163) presenting the results of phosphorylation of threonine 160 on the conformation switching of the whole CDK2 structure

\section{Evolution of the System and Future Research}

Searching information in the distributed bioinformatics databases takes integral part of the signal cascade analysis process. The paper presents the simulation system under development in the Institute of Informatics, Silesian University of Technology, Gliwice, Poland intended to signal transduction simulation using the data from BIND, BioCarta, PDB (Rutgers) databases, and others. The considered system includes the algorithms of fused signal cascades analysis, the nanostructures conformation determination, the sequence (amino acids chain) and shape (conformation) similarity searching and the solution of the encapsulation of databases (DB mirrors, interfaces and control) enables to use the proper database in the on-line computing.

\section{References}

1. Berman, H.M., Westbrook, J., Feng, Z., Gilliland, G., Bhat, T.N., Weissig, H., Shindyalov, I.N., Bourne, P.E.: The Protein Data Bank. Nucleic Acids Res. 28 (2000), 235-242

2. Greer, D.S., Westbrook, J.D., Bourne, P.E.: An ontology driven architecture for derived representations of macromolecular structure. Bioinformatics. 18 (2002) 1280-1281

3. Apweiler, R., Bairoch, A., Wu, C.H., et al.: UniProt: the Universal Protein knowledgebase. Nucleic Acids Research. 32 (2004) D115-D119 
4. Berridge, M.J.: The Molecular Basis of Communication within the Cell. Scientific American. 253 (4) (1985) 142-152

5. Bourne, P.E., Berman, H.M., Watenpaugh, K., Westbrook, J.D., Fitzgerald, P.M.D.: The macromolecular Crystallographic Information File (mmCIF). Methods Enzymol., 277 (1997) 571-590

6. Brown, N.R. et al.: Effects of Phosphorylation of Threonine 160 on Cyclin-dependent Kinase 2 Structure and Activity. J. of Biol. Chem. 274(13) (1999) 8746-8756

7. Znamirowski, L., Zukowska, E.D.: Simulation of Post-translational Conformations in the Ribosomal Polypeptide Synthesis. Proc. of the IASTED Intern. Conf. Modeling and Simulation, Marina del Rey, California, ACTA Press, Anaheim-Calgary-Zurich (2002) 97-102

8. Benson, D.A., Karsch-Mizrachi, I., Lipman, D.J., Ostell, J., Wheeler, D.L.: GenBank: update. Nucleic Acids Res. 32(Database issue) (2004) D23-6

9. BioCarta: Charting Pathways of Life. http://www.biocarta.com/genes/

10. Sayle R. RasMol: Molecular Graphics Visualization Tool. Biomolecular Structures Group, Glaxo Wellcome Research \& Development, Stevenage, Hartfordshire 1998, H. J. Bernstein. v.2.7.1.1, rasmol@bernstein-plus-sons.com

11. Boeckmann, B., Bairoch, A., Apweiler, R., et al.: The SWISS-PROT protein knowledgebase and its supplement TrEMBL in 2003. Nucleic Acids Res. 31 (2003) 365-370

12. Bader, G.D., Betel, D., Hogue, C.W.V.: BIND: the Biomolecular Interaction Network Database. Nucleic Acids Research. Vol. 31(1) (2003) 248-250

13. Snyder S.H.: The Molecular Basis of Communication between Cells. Scientific American, 253 (4) (1985) 132-141, (1985)

14. Signal Transduction Simulation System Using the Distributed Databases Environment. Research Project BW/2004/05, Institute of Informatics, Silesian University of Technology, Gliwice (2005)

15. http://www.omg.org

16. Warecki, S., Znamirowski, L.: Random Simulation of the Nanostructures Conformations, Intern. Conference on Computing, Communication and Control Technology, Proceedings Volume I, The Intern. Institute of Informatics and Systemics, Austin, Texas, August 14-17, p. 388-393, (2004)

17. Callaway, J., Cummings, M., et al.: Protein Data Bank Contents: Atomic Coordinate Entry Format Description, Federal Govern. Agency. (1996) http://www.rcsb.org/pdb/docs/format/

18. http://openmms.sdsc.edu

19. Tonegawa, S.: The Molecules of the Immune System, Scientific American, 253 (4) (1985) 122-131

20. Wang, Y., Addess, K.J., Geer, L., Madej, T., Marchler-Bauer, A., Zimmerman, D., Bryant S.H.: MMDB: 3D structure data in Entrez. Nucleic Acids Res. 28 (2000) 243-245

21. Ray, L.B.,: The Science of Signal Transduction. Science. 284 (1999) 755-756

22. Znamirowski, L.: Switching VLSI Structures. Reprogrammable FPAA Structures. Nanostructures. Studia Informatica. Vol. 25 (4A) (60) (2004) 1-236

23. Ponder, J.: Tinker - Software Tools for Molecular Design. Dept. of Biochemistry \& Molecular Biophysics, Washington University, School of Medicine, St. Louis (2001)

24. van Helden, J., Naim, A., Mancuso, R., et al.: Representing and analysing molecular and cellular function using the computer. Biol Chem. 381(9-10) (2000) 921-35

25. Bellman, R.: Dynamic Programming. Princeton University Press, Princeton, N. J. (1957)

26. Metropolis, N., Ulam, S.: The Monte Carlo Method, Journal of the American Stat. Assoc., 44 (247) (1949) 335-341, (1949) 